Research Paper

\title{
Combination analysis of NOS3, ABCB1 and IL23R polymorphisms with alcohol-induced osteonecrosis of the femoral head risk in Chinese males
}

\author{
Yuan Wang ${ }^{1, *}$, Xuejun Yang ${ }^{2,3, *}$, Jianping Shi ${ }^{3}$, Yan Zhao ${ }^{2,3}$, Linlin Pan ${ }^{2,3}$, Jinqiu \\ Zhou $^{4}$, Guoqiang Wang ${ }^{2,3}$ and Jianzhong Wang ${ }^{2,3}$ \\ ${ }^{1}$ Department of Orthopedics, the People's Hospital of Manzhouli City, Manzhouli 021400, Inner Mongolia, China \\ ${ }^{2}$ Department of Orthopedics, the Second Affiliated Hospital, Inner Mongolia Medical University, Hohhot 010030, Inner \\ Mongolia, China \\ ${ }^{3}$ The College of Traditional Chinese Medicine, Inner Mongolia Medical University, Hohhot 010010, Inner Mongolia, China \\ ${ }^{4}$ Department of Endocrine, the 253th Hospital of People's Liberation Army of China, Hohhot 010010, Inner Mongolia, China \\ *The first two authors should be regarded as joint first authors \\ Correspondence to: Jianzhong Wang, email: wangjianzhong3013@126.com \\ Guoqiang Wang, email: wangguoqiang3013@126.com \\ Keywords: NOS3, ABCBI, IL23R, osteonecrosis of the femoral head, gene polymorphism \\ Received: July 10, $2016 \quad$ Accepted: March 16, $2017 \quad$ Published: April 04, 2017 \\ Copyright: Wang et al. This is an open-access article distributed under the terms of the Creative Commons Attribution License \\ (CC-BY), which permits unrestricted use, distribution, and reproduction in any medium, provided the original author and source \\ are credited.
}

\section{ABSTRACT}

Background: Common variants of multiple genes played a crucial role in osteonecrosis of the femoral head (ONFH) onset which was proved by many previous reports. We hypothesized that polymorphisms in NOS3, ABCB1 and IL23R were related to individual differences in alcohol sensitivity and the development of alcohol-induced ONFH.

Methods: In this case-control study, we evaluated 8 SNPs in three genes in the Chinese Han population including 355 male cases and 355 healthy male controls. These SNPs were genotyped by Sequenom MassARRAY RS1000. To identify their relationship with alcohol-induced ONFH susceptibility using $\chi^{2}$ test and genetic model analysis.

Results: We found an association with alcohol-induced ONFH susceptibility for 4 SNPs (rs743506, rs3918184, rs13233308 and rs6693831) in three genes after adjusted by age. The genotype "G/A" of rs743506 in NOS3 gene acts as a risk factor in genotype $(P=0.003)$, dominant $(P=0.048)$, recessive $(P=0.005)$ and additive model $(P=0.006)$; The genotype "T/C" of rs3918184 in NOS3 gene acts as a risk factor in genotype $(P=0.012)$ and recessive model $(P=0.009)$; The genotype "T/C" of rs13233308 in $A B C B 1$ gene acts as a risk factor in genotype $(P=0.038)$ and additive model $(P=0.041)$; The genotype "T/C" of rs6693831 in IL23R gene acts as a protective factor in genotype model $(P=0.046)$.

Conclusions: This study provides evidence for three alcohol-induced ONFH susceptibility genes (NOS3, ABCB1 and IL23R) in Chinese males and polymorphisms of them may be associated with alcohol-induced ONFH risk.

\section{INTRODUCTION}

Osteonecrosis of the femoral head (ONFH) indicates a debilitating disorder that the bone collapses, happening in a special anatomical site of femoral head and resulting in osteocyte death $[1,2]$. It mainly affects younger males who is between 30 and 50 years old. In China, there are seven million patients with osteonecrosis and be found annually new patients to increase by 150,000 to 200,000 patients [3]. The seventy-five to ninety percent cases of ONFH have been associated with various risk factors, involving alcohol 
Table 1: Characteristics of 355 alcohol-induced ONFH male subjects

\begin{tabular}{llll}
\hline Parameters & Cases & Controls & P value \\
\hline Age $[$ mean \pm SD $]$ & $44.91 \pm 9.85$ & $46.02 \pm 9.61$ & NS \\
Sex $[$ male] & 355 & 355 & \\
BMI $\left(\mathrm{kg} / \mathrm{m}^{2}\right)$ & & & NS \\
$\geq 25$ & 53 & 61 & NS \\
$<25$ & 302 & 294 & \\
\hline
\end{tabular}

SD, standard deviation: NS, notsignificant: BMI, body mass index.

abuse, corticosteroid use, hip trauma and smoking [4]. Increasing of adipose vesicles in the blood circulating is induced these risk factors, adding lipid deposition in osteocytes of the femoral head and resulting in embolism, which influence finally blood flow [5].

Current evidence suggests that being related to the risk of ONFH and eight SNPs in three genes of our research can be identified through the genome-wide association studies $[1,6,7]$. Nitric oxide is synthesised from L-arginine and has three isoforms of synthases (NOS): endothelial (eNOS) neuronal (nNOS) and inducible(iNOS). The eNOS is expressed in normal adult bone as a constitutive isoform [8]. A previous study in Korean patients indicated that idiopathic osteonecrosis of the femoral head was significantly associated with polymorphism of eNOS gene, but it also indicate that polymorphism in intron 4 of eNOS was not significant differences [9]. Glueck et al. found that the pathogenesis of idiopathic ONFH are related with the intron 4 of eNOS polymorphism and synthetic loss of nitric oxide outcome in African American and Caucasian patients. The ABCB1, is also called adenosine triphosphatebinding cassette $\mathrm{B} 1$, can encode the transport protein and made an significant influence on distribution and absorption in the mammalian body [10]. So far a few single nucleotide polymorphisms (SNPs) of $A B C B 1$ gene have been identified, of which mutations in exon 21 (G2677T) and exon 26 (C3435T) are associated with alteration of P-gp expression or function, as recently reviewed by Sakaeda et al. and Fromm et al [11, 12]. Interleukin 23 receptor (IL23) is a proinflammatory cytokine. According to previous reports indicated it inhibited osteoclastogenesis by Receptor Activator for Nuclear Factor $\kappa \mathrm{B}$ Ligand with $\mathrm{T}$ cell affection [13]. Kim et al. discover polymorphisms in the Interleukin 23 receptor $(I L 23 R)$ gene are associated with $\mathrm{ONFH}$ in Korean population [14].

A few studies investigated the association of NOS3, $A B C B 1$ and $I L 23 R$ polymorphism with the pathogenesis of ONFH. We hypothesized that NOS3, ABCBI and IL23R genes have crucial roles in the development of alcoholinduced ONFH. To identify whether polymorphisms of these genes to be associated with alcohol-induced ONFH in Chinese males.

\section{RESULTS}

In the current study, a total of 355 male cases (median age at diagnosis $44.91 \pm 9.85$ years) and 355 healthy male controls (median age $46.02 \pm 9.61$ years) were included. The basic characteristics of cases and controls were showed in Table 1. The primers of the 8 candidate SNPs are revealed in Table 2. Eight SNP loci(rs6693831, rs790631, rs4148749, rs10808072, rs13233308, rs3918227, rs3918184 and rs743506) in the NOS3, $A B C B 1$ and $I L 23 R$ were evaluated in this study. SNP ID, HWE P value, allele A/B, MAF control/ case, odds ratio, $95 \%$ confidence interval and $\mathrm{P}$ value were listed in Table 3. Using $\mathrm{x}^{2}$ test, we found significant differences in frequency of alleles and rs13233308 in the $A B C B 1$ gene and rs743506 in the NOS3 gene were associated with increased risk of alcohol-induced ONFH risk by allele model analysis (rs13233308, $P=0.032$, odds ratio [OR]: $1.26,95 \%$ confidence interval [CI]: 1.02-1.55 and rs $743506, P=0.006$, OR: $1.39,95 \%$ CI: $1.09-1.78)$. All of the SNPs were in Hardy-Weinberg equilibrium (HWE) in the control population of this study.

In this study, the whole of patients were male and we then further analyzed the association of the NOS3, ABCB1 and IL23R gene polymorphisms with alcohol-induced ONFH patients adjusted by age in Table 4 . The "G/A" of rs743506 increased alcohol-induced ONFH risk was found by the genotype model $(P=0.004, \mathrm{OR}, 2.57 ; 95 \% \mathrm{CI}, 1.36$ 4.86 crude; $P=0.003$, OR, 2.66; $95 \%$ CI, 1.40-5.05 adjusted by age), the dominant model ( $P=0.048, \mathrm{OR}, 1.35 ; 95 \% \mathrm{CI}$, $1.01-1.82$ adjusted by age), the recessive model ( $P=0.006$, OR, 2.40; 95\% CI, 1.28-4.49 crude; $P=0.005$, OR, 2.48; $95 \%$ CI, $1.32-4.65$ adjusted by age) and the additive model $(P=0.008$, OR, $1.38 ; 95 \%$ CI, $1.09-1.75$ crude; $P=0.006$, OR, 1.39; 95\% CI, 1.10-1.78 adjusted by age), analysis respectively. The "T/C" of rs3918184 increased alcoholinduced ONFH risk was found by the genotype model $(P=$ 0.016 , OR, $1.86 ; 95 \%$ CI, $1.12-3.08$ crude; $P=0.012$, OR, $1.91 ; 95 \%$ CI, $1.15-3.18$ adjusted by age) and the recessive model $(P=0.013$, OR, $1.85 ; 95 \%$ CI, $1.14-2.99$ crude; $P$ $=0.009$, OR, 1.89; $95 \%$ CI, 1.17-3.08 adjusted by age), analysis respectively. The "T/C" of rs13233308 increased alcohol-induced ONFH risk was showed by the genotype model $(P=0.031$, OR, $1.59 ; 95 \%$ CI, $1.04-2.43$ crude; $P=$ 0.038 , OR, $1.57 ; 95 \%$ CI, $1.03-2.39$ adjusted by age), the 
Table 2: Polymerase chain reaction primers of selected SNPs

\begin{tabular}{|c|c|c|c|}
\hline SNP ID & 1st - PCR primer sequences & 2nd - PCR primer sequences & UEP sequences \\
\hline rs6693831 & $\begin{array}{c}\text { ACGTTGGATGGTTACGGTC } \\
\text { ACCTTGGAAAG }\end{array}$ & $\begin{array}{l}\text { ACGTTGGATGCTCAT } \\
\text { AACCAGAAGATTCCC }\end{array}$ & TTCCCATGTGGGAAAGTTC \\
\hline rs790631 & $\begin{array}{c}\text { ACGTTGGATGGTCTTATTAG } \\
\text { GATAAAACCCC }\end{array}$ & $\begin{array}{c}\text { ACGTTGGATGCCTTTT } \\
\text { GCATATGCAGAAT }\end{array}$ & CACAAATACAATTCTCAAGTC \\
\hline rs4148749 & $\begin{array}{c}\text { ACGTTGGATGACAGTCCCA } \\
\text { CTTGGATAAAG }\end{array}$ & $\begin{array}{c}\text { ACGTTGGATGACAGAT } \\
\text { GACACCACTTGGAG }\end{array}$ & attACACCACTTGGAGACCATATTTA \\
\hline rs10808072 & $\begin{array}{c}\text { ACGTTGGATGCCTTTGTAA } \\
\text { CTTTCCCAATG }\end{array}$ & $\begin{array}{c}\text { ACGTTGGATGCCTGAA } \\
\text { AATGTTGTGTGTTGG }\end{array}$ & ggggTTGAGAATTGTATTGCTAGTTA \\
\hline rs 13233308 & $\begin{array}{c}\text { ACGTTGGATGCTGTTTCAAT } \\
\text { GATCCAGGTG }\end{array}$ & $\begin{array}{c}\text { ACGTTGGATGGTTG } \\
\text { GTGCTACCCTCAAAAT }\end{array}$ & cttcGCTACCCTCAAAATATATCCA \\
\hline rs3918227 & $\begin{array}{c}\text { ACGTTGGATGTGAGTGCCG } \\
\text { TTCATTGTGTG }\end{array}$ & $\begin{array}{l}\text { ACGTTGGATGTTCAT } \\
\text { AATAGCCCCGACCTG }\end{array}$ & gGTCACCAACAAGAGAATG \\
\hline rs3918184 & $\begin{array}{c}\text { ACGTTGGATGCCATCGAGA } \\
\text { AACATTACCCG }\end{array}$ & $\begin{array}{l}\text { ACGTTGGATGCTTGA } \\
\text { ATCCCTGACCTCAGC }\end{array}$ & gggagTACAGGCGTGAGCCACCA \\
\hline rs 743506 & $\begin{array}{c}\text { ACGTTGGATGGAGCAAGC } \\
\text { TAGATTGCTAGG }\end{array}$ & $\begin{array}{l}\text { ACGTTGGATGAAATG } \\
\text { CACCCCCACCAAAAG }\end{array}$ & teccgCCСТCTGGGCTCСТСТCC \\
\hline
\end{tabular}

PCR, polymorphism chain reaction; SNP, single-nucleotide polymorphism; UEP, unextended mini-sequencing primer.

Table 3: Allele frequencies of polymorphic variants of the candidate genes in this study

\begin{tabular}{|c|c|c|c|c|c|c|c|c|c|}
\hline \multirow[b]{2}{*}{ SNP ID } & \multirow[b]{2}{*}{ Chromosome } & \multirow[b]{2}{*}{ Position } & \multirow[b]{2}{*}{ Gene } & \multirow[b]{2}{*}{$\begin{array}{c}\text { HWE } \\
P \text { value }\end{array}$} & \multirow[b]{2}{*}{$\begin{array}{c}\text { Alleles } \\
\text { A/B }\end{array}$} & \multirow[b]{2}{*}{$\begin{array}{c}\text { MAF } \\
\text { control }\end{array}$} & \multirow[b]{2}{*}{$\begin{array}{c}\text { MAF } \\
\text { case }\end{array}$} & \multicolumn{2}{|c|}{ Allele model } \\
\hline & & & & & & & & OR $(95 \%$ CI) & $\begin{array}{c}P \\
\text { value }\end{array}$ \\
\hline rs6693831 & 1 & 67720867 & $I L 23 R$ & 0.1227 & $\mathrm{~T} / \mathrm{C}$ & 0.254 & 0.238 & $0.91(0.72-1.17)$ & 0.48 \\
\hline rs790631 & 1 & 67676922 & $I L 23 R$ & 0.5857 & $\mathrm{C} / \mathrm{T}$ & 0.049 & 0.061 & $1.24(0.79-1.96)$ & 0.35 \\
\hline rs4148749 & 7 & 87144413 & $A B C B 1$ & 0.5719 & $\mathrm{C} / \mathrm{G}$ & 0.17 & 0.163 & $0.95(0.72-1.26)$ & 0.72 \\
\hline rs10808072 & 7 & 87176463 & $A B C B 1$ & 0.4747 & $\mathrm{G} / \mathrm{A}$ & 0.341 & 0.338 & $0.99(0.79-1.23)$ & 0.91 \\
\hline rs 13233308 & 7 & 87244960 & $A B C B 1$ & 0.9156 & $\mathrm{~T} / \mathrm{C}$ & 0.497 & 0.554 & $1.26(1.02-1.55)$ & $0.032 *$ \\
\hline rs3918227 & 7 & 150700946 & NOS3 & 0.6408 & $\mathrm{~A} / \mathrm{C}$ & 0.063 & 0.063 & $1(0.65-1.53)$ & 1 \\
\hline rs3918184 & 7 & 150702219 & NOS3 & 0.7983 & $\mathrm{~T} / \mathrm{C}$ & 0.292 & 0.339 & $1.25(0.99-1.56)$ & 0.05 \\
\hline rs743506 & 7 & 150706915 & NOS3 & 0.6418 & $\mathrm{G} / \mathrm{A}$ & 0.218 & 0.280 & $1.39(1.09-1.78)$ & $0.006 *$ \\
\hline
\end{tabular}

OR, odds ratio, $95 \% \mathrm{CI}, 95 \%$ confidence interval. ${ }^{*} P<0.05$, statistical significance.

$\mathrm{A} / \mathrm{B}$ stands for minor/major alleles on the control sample frequencies.

SNPs are excluded at HWE $P<0.05$.

dominant model ( $P=0.049$, OR, $1.43 ; 95 \%$ CI, $1.00-2.04$ crude) and the additive model $(P=0.033, \mathrm{OR}, 1.26 ; 95 \%$ CI, 1.02-1.55 crude; $P=0.041$, OR, $1.25 ; 95 \%$ CI, $1.01-1.54$ adjusted by age), analysis respectively. Meanwhile, we also found the "T/C" of rs6693831 decrease alcohol - induced ONFH risk by the genotype model $(P=0.041$, OR, 0.72 ; 95\% CI, 0.53-0.98 crude; $P=0.046$, OR, $0.73 ; 95 \%$ CI, $0.53-0.99$ adjusted by age).

The results of the association between the NOS3 haplotype and alcohol-induced ONFH risk were listed in Table 5. Haplotype "CT" in Block 1 was found to be associated with increased alcohol-induced ONFH risk $(P$ $=0.044$, OR, $1.26 ; 95 \%$ CI, 1.01-1.58 adjusted by age) (Figure 1).

\section{DISCUSSION}

Non-traumatic ONFH has been diffusely awaked as a pathological define with numerous etiologies, the precise 
Table 4: Association between single-nucleotide polymorphisms and risk of alcohol-induced ONFH based on logistic test

\begin{tabular}{|c|c|c|c|c|c|c|}
\hline SNP ID & Model & Genotype & OR $(95 \%$ CI) & $p$ value & †OR $(95 \% \mathrm{CI})$ & $\dagger p$ value \\
\hline \multirow[t]{4}{*}{ rs6693831 } & Genotype & $\mathrm{T} / \mathrm{C}$ & $0.72(0.53-0.98)$ & $0.041 *$ & $0.73(0.53-0.99)$ & $0.046^{*}$ \\
\hline & Dominant & $\mathrm{T} / \mathrm{C}$ & $0.79(0.59-1.07$ & 0.121 & $0.80(0.59-1.08)$ & 0.150 \\
\hline & Recessive & $\mathrm{T} / \mathrm{C}$ & $1.57(0.83-2.94)$ & 0.163 & $1.67(0.88-3.15)$ & 0.120 \\
\hline & Additive & $\mathrm{T} / \mathrm{C}$ & $0.92(0.72-1.17)$ & 0.493 & $0.93(0.73-1.19)$ & 0.570 \\
\hline \multirow[t]{4}{*}{ rs790631 } & Genotype & $\mathrm{C} / \mathrm{T}$ & $2.05(0.18-2.27)$ & 0.560 & $1.96(0.17-2.18)$ & 0.582 \\
\hline & Dominant & $\mathrm{C} / \mathrm{T}$ & $1.23(0.76-1.99)$ & 0.393 & $1.26(0.77-2.03)$ & 0.356 \\
\hline & Recessive & $\mathrm{C} / \mathrm{T}$ & $2.01(0.81-2.22)$ & 0.571 & $1.92(0.17-2.13)$ & 0.593 \\
\hline & Additive & $\mathrm{C} / \mathrm{T}$ & $1.24(0.78-1.94)$ & 0.358 & $1.25(0.79-1.97)$ & 0.328 \\
\hline \multirow[t]{4}{*}{ rs4148749 } & Genotype & $\mathrm{C} / \mathrm{G}$ & $0.74(0.31-1.79)$ & 0.511 & $0.74(0.31-1.78)$ & 0.498 \\
\hline & Dominant & $\mathrm{C} / \mathrm{G}$ & $0.97(0.71-1.34)$ & 0.871 & $0.98(0.71-1.35)$ & 0.902 \\
\hline & Recessive & $\mathrm{C} / \mathrm{G}$ & $0.74(0.31-1.78)$ & 0.507 & $0.73(0.31-1.77)$ & 0.492 \\
\hline & Additive & $\mathrm{C} / \mathrm{G}$ & $0.95(0.72-1.25)$ & 0.724 & $0.95(0.72-1.26)$ & 0.745 \\
\hline \multirow[t]{4}{*}{ rs 10808072} & Genotype & $\mathrm{G} / \mathrm{A}$ & $0.99(0.56-1.57)$ & 0.812 & $0.96(0.57-1.61)$ & 0.874 \\
\hline & Dominant & $\mathrm{G} / \mathrm{A}$ & $1.01(0.74-1.35)$ & 0.983 & $1.01(0.74-1.35)$ & 0.982 \\
\hline & Recessive & $\mathrm{G} / \mathrm{A}$ & $0.93(0.57-1.51)$ & 0.774 & $0.95(0.58-1.55)$ & 0.845 \\
\hline & Additive & $\mathrm{G} / \mathrm{A}$ & $0.98(0.78-1.23)$ & 0.906 & $0.99(0.79-1.24)$ & 0.941 \\
\hline \multirow[t]{4}{*}{ rs13233308 } & Genotype & $\mathrm{T} / \mathrm{C}$ & $1.59(1.04-2.43)$ & $0.031 *$ & $1.57(1.03-2.39)$ & $0.038^{*}$ \\
\hline & Dominant & $\mathrm{T} / \mathrm{C}$ & $1.43(1.00-2.04)$ & $0.049 *$ & $1.41(0.99-2.01)$ & 0.060 \\
\hline & Recessive & $\mathrm{T} / \mathrm{C}$ & $1.29(0.93-1.81)$ & 0.124 & $1.29(0.92-1.79)$ & 0.140 \\
\hline & Additive & $\mathrm{T} / \mathrm{C}$ & $1.26(1.02-1.55)$ & $0.033 *$ & $1.25(1.01-1.54)$ & $0.041 *$ \\
\hline \multirow[t]{4}{*}{ rs3918227 } & Genotype & $\mathrm{A} / \mathrm{C}$ & $1.00(0.17-7.14)$ & 1.000 & $1.06(0.14-7.59)$ & 0.955 \\
\hline & Dominant & $\mathrm{A} / \mathrm{C}$ & $1.00(0.63-1.57)$ & 1.000 & $1.01(0.64-1.58)$ & 0.971 \\
\hline & Recessive & $\mathrm{A} / \mathrm{C}$ & $1.00(0.14-7.13)$ & 1.000 & $1.06(0.15-7.17)$ & 0.955 \\
\hline & Additive & $\mathrm{A} / \mathrm{C}$ & $1.00(0.65-1.52)$ & 1.000 & $1.01(0.66-1.54)$ & 0.963 \\
\hline \multirow[t]{4}{*}{ rs3918184 } & Genotype & $\mathrm{T} / \mathrm{C}$ & $1.86(1.12-3.08)$ & $0.016^{*}$ & $1.91(1.15-3.18)$ & $0.012 *$ \\
\hline & Dominant & $\mathrm{T} / \mathrm{C}$ & $1.15(0.86-1.55)$ & 0.346 & $1.16(0.86-1.56)$ & 0.322 \\
\hline & Recessive & $\mathrm{T} / \mathrm{C}$ & $1.85(1.14-2.99)$ & $0.013 *$ & $1.89(1.17-3.08)$ & $0.009 *$ \\
\hline & Additive & $\mathrm{T} / \mathrm{C}$ & $1.23(0.99-1.54)$ & 0.061 & $1.25(0.99-1.55)$ & 0.051 \\
\hline \multirow[t]{4}{*}{ rs743506 } & Genotype & $\mathrm{G} / \mathrm{A}$ & $2.57(1.36-4.86)$ & $0.004 *$ & $2.66(1.40-5.05)$ & $0.003^{*}$ \\
\hline & Dominant & $\mathrm{G} / \mathrm{A}$ & $1.33(0.99-1.79)$ & 0.058 & $1.35(1.01-1.82)$ & $0.048 *$ \\
\hline & Recessive & $\mathrm{G} / \mathrm{A}$ & $2.40(1.28-4.49)$ & $0.006^{*}$ & $2.48(1.32-4.65)$ & $0.005^{*}$ \\
\hline & Additive & $\mathrm{G} / \mathrm{A}$ & $1.38(1.09-1.75)$ & $0.008 *$ & $1.39(1.10-1.78)$ & $0.006^{*}$ \\
\hline
\end{tabular}

OR, odds ratio; $95 \% \mathrm{CI}, 95 \%$ confidence interval;

$* P<0.05$, statistical significance. $\dagger$ Adjusted by age.

Table 5: Haplotype analysis results of rs3918227 and rs3918184 in NOS3 gene

\begin{tabular}{lcccc}
\hline Haplotype & freq(case) & freq(control) & $\dagger$ OR(95\%CI) & $\dagger P$ \\
\hline CT & 0.340 & 0.293 & $1.26(1.01-1.58)$ & $0.044^{*}$ \\
AC & 0.063 & 0.063 & $1.12(0.72-1.73)$ & 0.610 \\
\hline
\end{tabular}

Freq, frequency; OR, odd ratio; CI, confidence interval.

$* P<0.05$, statistical significance: $\uparrow$ Adjusted by age 
pathogenesis of osteonecrosis leaves to be evaluated. Alcoholism and chronic steroid use were considered to be the essential risk factors induced osteonecrosis. Genetic mutation of alcohol-metabolizing enzyme genes has been associated with alcohol-induced osteonecrosis and polymorphism of $N O S 3, A B C B 1$ and $I L 23 R$ gene are related with developing osteonecrosis [6, 15-17].

The NOS3 gene located in chromosome $7 \mathrm{q} 36.1$ involves two SNPs ("G/A" in rs743506 and "T/C" in rs3918184) related with increased alcohol-induced ONFH risk. Endothelial nitric oxide synthase is the remarkable NOS isoform expressed in normal bone and eNOS adjusts bone resorption and formation and oestrogen are dependent on NO production via eNOS in the skeletal system [18]. Excessive NO production was found in various rheumatic diseases, including rheumatoid arthritis, systemic lupus erythematosus, osteoarthritis and vasculitis [8]. Güler $\mathrm{S}$ et al. found the genotype "GA" of rs743506 showed risk effect was associated with aura in migraine patients [19]. It proved that mutation of rs743506 would affect function of NO in cerebral blood flow regulation and include the activation of nociceptors in the vascular system and the release of vasoactive neuropeptides in the neurogenic inflammatory response [20]. Meanwhile, Juan P et al. found genotype "T/C" of rs3918184 be related with increased risk of hypertension, preeclampsia and stroke [21]. Combination with our research, rs743506 and rs3918184 associated with increased with alcoholinduced ONFH risk and it may also be effect NO in the femoral head blood flow regulation. The NOS is fundamentally expressed in vascular endothelium and had been proved to shorten NO norms in human plasma [22]. Our study suggests that the genotype "GA" of rs743506 and genotype "T/C" of rs3918184 polymorphisms may be risk factors for alcohol-induced ONFH, and NO brought in fundamentally decided eNOS may make a significant effect on the pathogenesis of alcohol-induced ONFH.

The $A B C B 1$ gene, also known as Multidrugresistant transporter-1(MDRl), was located in chromosome 7q21.12 and the genotype "T/C" of rs13233308 related with increased alcohol-induced ONFH risk. This gene spans $4.5 \mathrm{~kb}$ encoding a 1280

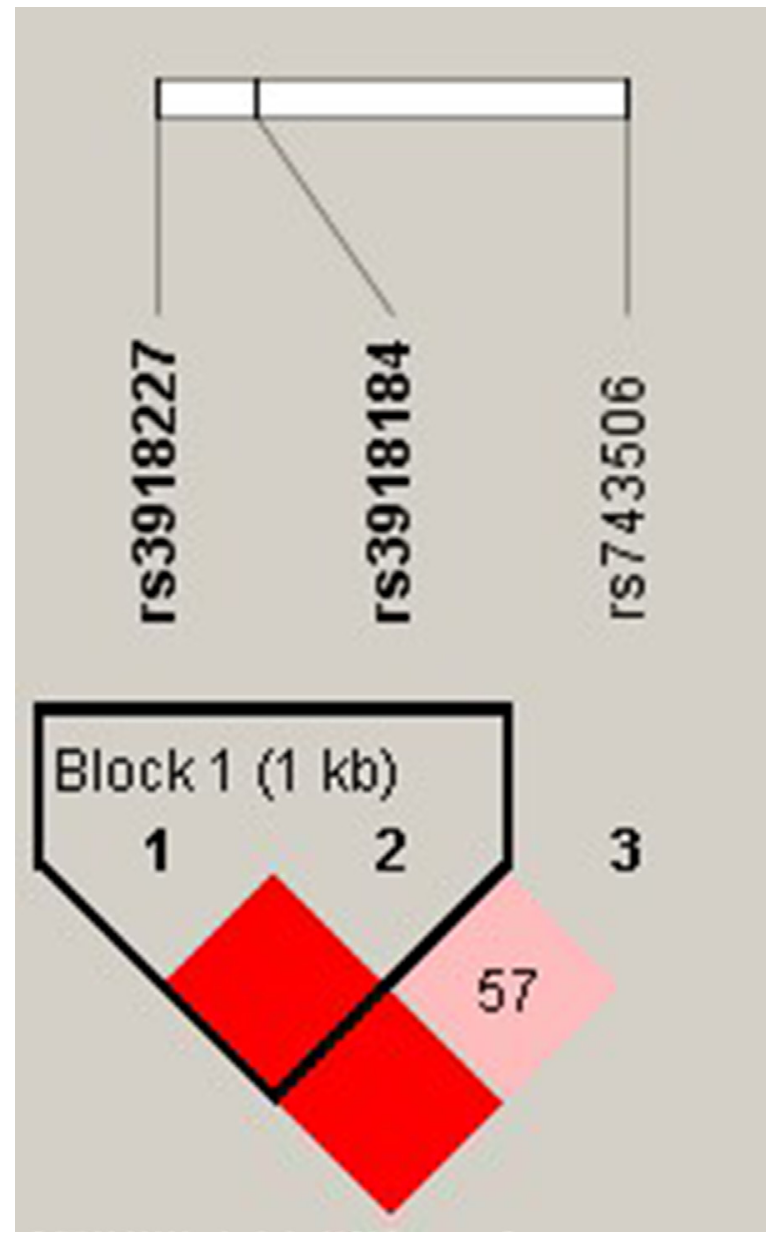

Figure 1: Haplotype-Block Map for NOS3 Based on rs3918227, rs3918184 and rs743506. Linkage disequilibrium (LD) among single nucleotide polymorphisms (SNPs) analyzed in chromosome 7q. LD is indicated by standard color schemes with bright red for very strong $L D\left(L D>2, D^{\prime}=1\right)$ and pink red $\left(L D>2, D^{\prime}<1\right)$ for intermediate $L D$. 
amino acid (aa) polypeptide. After glycosylation of the amino acid polypeptide, a $170-\mathrm{kDa}$ of protein named P-glycoprotein (P-gp) can be formed. P-gp is occurred in various tissue cells and is required for pumping lipophilic drugs (including glucocorticoids) out of the cells. This causes cytotoxic damage and the intracellular drug concentration to decrease is subsequently reduced. In addition, $\mathrm{P}-\mathrm{gp}$, encoded by the human $A B C B 1$ gene, is an important determinant in absorption, elimination of drugs and tissue targeting [23]. Xue et al. found polymorphisms of $A B C B 1$ gene be related with decreased risk of steroid-induced avascular necrosis of the femoral head in a Chinese population [16]. Zhang et al. also found $A B C B 1$ gene polymorphisms associated with decrease risk for steroid-induced osteonecrosis of the femoral head in Chinese population [1]. To date, 28 SNPs in the $A B C B 1$ gene have been reported at 27 positions in Caucasians and Africans [24, 25]. Apparently, these results was inconsistent with ours. Meanwhile, we must notice that ethnic differences may be considered to take an important effect to ABCB1 gene and ONFH. The homozygosity of both the wildtype and variant alleles of MDR1 (C1236 T, G2677T/A, and C3435T) in healthy control subjects was similar to that reported by other Asian studies, which were performed with healthy Chinese, Japanese, and Korean subjects. However, the allele frequencies were significantly higher than those observed in European ethnicities, such as German, Russian, and Serbian [16]. Thus, further studies related with in other ONFH populations are required to confirm and realize $A B C B 1$ gene different mechanisms in steroidinduced and alcohol-induced ONFH.

The IL23R gene was located in chromosome $1 \mathrm{p} 31.3$ and the genotype "T/C" of rs6693831 related with decreased alcohol-induced ONFH risk. Interleukin 23 receptor, a heterodimer protein, was composed of two subunits: IL-23R and IL-12Rb1. IL-23R, expressed on activated $\mathrm{T}$ cells, dendritic cells, macrophages and natural killer (NK) cells, binds particularly to IL-23, produced primarily by dendritic cells and activated macrophages from peripheral tissues [26]. Kim et al. found polymorphisms of the $I L 23 R$ gene were expressively related with ONFH among the Korean population. Among others, the polymorphism of the IL23R gene were particularly related with idiopathic ONFH risk. However, there was no significant associated with alcohol-induced and steroid-induced ONFH [14]. Apparently, this results were inconsistent compared with our results. In our study, rs6693831 of $I L 23 R$ gene were related with decrease alcohol-induced ONFH risk in Chinese males. Alcoholism, which can influence fat metabolism and backlogging cell pressure, which considers absorption of the blood flood, were considerable risk factors with osteonecrosis [27]. We may thought whether the effect of alcohol abuse was stronger than genetic factors in regards to alcohol- induced ONFH patients. Meanwhile, the difference of results must be considered the effect related ethnic. In further studies, we need larger different ethnic populations to verify our results.

In this case-control study, some limitations were intrinsic and must be marked. First, to avoid selection bias, alcohol-induced ONFH cases and healthy controls came from the same hospital. However, this bias not necessarily to be of consequence because of demographic variables. Secondly, the sample size (355 male cases and 355 male controls) is not large enough in our work. We performed a power analysis and just found the power of rs743506 in NOS3 gene was 0.91. The power of seven surplus SNPs was $<0.75$. We considered that the number of samples was small in association studies and confirming our finding required a larger sample size [28].

Our research provides a new evidence for a relationship between $N O S 3, A B C B 1$ and $I L 23 R$ gene and alcohol-induced ONFH in Chinese males onset, which may shed light on the etiology of alcohol-induced ONFH. Functional studies are further required to evaluate the correlation of genotype and phenotype in a large cohort of various ethnicities.

\section{MATERIALS AND METHODS}

\section{Ethics statement}

The use of the protocol in this study was strictly affirmed to the principles expressed in the Declaration of Helsinki and were approved by the Ethical Committee of Zhengzhou Traditional Chinese Medicine Traumatology Hospital. Informed consent was signed from all of the participants.

\section{Study participants}

Three hundred and fifty male patients with alcoholinduced ONFH were consecutively enrolled in this study at the Zhengzhou Traditional Chinese Medicine Traumatology Hospital from June 2014 to January 2016. Patients with a history of ethanol consumption of at least $400 \mathrm{ml}$ per week were categorized under alcohol-induced osteonecrosis [29]. The development of alcohol-induced ONFH was diagnosed according to assessment by X-rays, magnetic resonance imaging (MRI), and bone scans [2]. The ONFH was present in one hip in 135 patients and in both hips in 220 patients (440 hips). Patients with a demonstrable history of direct trauma or with the possibility of a combination of causes were excluded.

We also enrolled 355 healthy male controls between July 2014 and January 2016 based on medical examination at Zhengzhou Traditional Chinese Medicine Traumatology Hospital. The controls had a history of ethanol consumption of at least $400 \mathrm{ml}$ per week, however, 
they had no alcohol-induced ONFH and other related diseases, no history of thromboembolic events and no symptoms of hip disease. All participants were restricted to Chinese Han population who lived in Zhengzhou city and its surrounding areas.

\section{Genotyping}

Genomic DNA was extracted and purified from the whole blood of all the participants using a kit (GoldMag, China) and their concentration was measured by spectrometry (DU530UV/VIS spectrophotometer; Beckman Instruments, Fullerton, CA). All PCR reactions were operated in a $25 \mathrm{ul}$ volume containing $10 \mathrm{pmol}$ of each primer, 1X PCR buffer, 10mM dNTP, $25 \mathrm{ng}$ of genomic DNA, and $1 \mathrm{U}$ of Taq DNA polymerase (Solgent, Daejeon, Korea). The conditions of PCR amplification were as follows: initial denaturation at $95^{\circ} \mathrm{C}$ for 15 min followed by 35 cycles of denaturation at $95^{\circ} \mathrm{C}$ for $20 \mathrm{sec}$, annealing at $55^{\circ} \mathrm{C}$ for $40 \mathrm{sec}$, extension at $72^{\circ} \mathrm{C}$ for $1 \mathrm{~min}$, and a final extension at $72^{\circ} \mathrm{C}$ for $5 \mathrm{~min}$. SNP genotyping was performed on the SEQUENOM MassARRAY_Analyzer 4 (Sequenom, Inc., San Diego, CA, USA) using genomic DNA in a single multiplex reaction. According to the manufacturer's instructions, the primers for polymerase chain reaction amplification and single base extension were designed by Sequenom Assay Design 3.0 software (Sequenom, San Diego, CA, USA)[30]. SNP genotyping using the standard protocol recommended by the manufacturer was performed by Sequenom MassARRAY RS1000. Data analyses and management were conducted by Sequenom Typer 4.0 Software [30, 31].

\section{Statistical analysis}

Microsoft Excel and SPSS 16.0 statistical package (SPSS, Chicago, IL) were used to perform statistical analyses. All $P$ values in this study were two-sided, and $P$ $=0.05$ was considered the threshold of whether statistical significance was achieved or not. A significant departure of genotype frequency from Hardy-Weinberg equilibrium (HWE) for each SNP was estimated using SNPStats (http://bioinfo.iconcologia.net/snpstats/start.htm). The genotype frequencies of cases and controls were calculated using $\chi 2$ test [32]. Odds ratios (ORs) and $95 \%$ confidence intervals $(95 \% \mathrm{CIs})$ were determined using unconditional logistic regression analysis with adjustment by age [33]. The four genetic models (Genotype, Dominant, Recessive and Additive) were applied using PLINK software (http://pngu.mgh.harvard.edu/purcell/plink/) to assess the association of SNPs with the risk of alcohol-induced ONFH.

\section{Abbreviations}

NOS3, nitric oxide synthase 3; ABCB1, adenosine triphosphate-binding cassette B1; IL23R, interleukin
23 receptor; PCR, polymerase chain reaction; UEP, unextended mini-sequencing primer; SNPs: single nucleotide polymorphisms; MAF: minor allele frequency; HWE: Hardy - Weinberg equilibrium; OR: Odds ratio; CI: confidence interval.

\section{Author contributions}

YW collected and analyzed the data, and drafted the manuscript; $X Y$ conceived the idea and critically revised the manuscript; JS and YZ collected and analyzed the data; LP and JZ participated in the design of the study and performed the statistical analysis. GW and JW participated in its design and coordination, and funded the study. All authors read and approved the final manuscript.

\section{ACKNOWLEDGMENTS}

This study was supported by grants from the National Natural Science Foundation of China (no. 81160228,81260284 and 81660378). We would also like to thank the hospital staff who contributed to the blood sample for this study.

\section{CONFLICTS OF INTEREST}

The authors declare that they have no competing interests.

\section{FUNDING}

This study was supported by grants from the National Natural Science Foundation of China (no. 81160228,81260284 and 81660378).

\section{REFERENCES}

1. Zhang Y, Kong X, Wang R, Li S, Niu Y, Zhu L, Chen W, Lin N. Genetic association of the P-glycoprotein gene $\mathrm{ABCB} 1$ polymorphisms with the risk for steroid-induced osteonecrosis of the femoral head in Chinese population. Molecular biology reports. 2014; 41:3135-3146.

2. Wang Y, Cao Y, Li Y, Guo Y, Wang Q, Yang M, Zhang $\mathrm{N}$, Jin T, Wang J. Genetic association of the ApoB and ApoA1 gene polymorphisms with the risk for alcoholinduced osteonecrosis of femoral head. International journal of clinical and experimental pathology. 2015; 8:11332-11339.

3. Yin JM, Liu Z, Zhao SC, Guo YJ, Liu ZT. Relationship between the Apolipoprotein AI, B gene polymorphism and the risk of non-traumatic osteonecrosis. Lipids in health and disease. 2014; 13:149.

4. Kaushik AP, Das A, Cui Q. Osteonecrosis of the femoral head: An update in year 2012. World journal of orthopedics. $2012 ; 3: 49-57$ 
5. Wang Y, Li Y, Mao K, Li J, Cui Q, Wang GJ. Alcoholinduced adipogenesis in bone and marrow: a possible mechanism for osteonecrosis. Clinical orthopaedics and related research. 2003; (410):213-224.

6. Zheng L, Wang W, Ni J, Li Z, Xiao T. The association of eNOS gene polymorphism with avascular necrosis of femoral head. PloS one. 2014; 9:e87583.

7. Kamiya S, Nakamura C, Fukawa T, Ono K, Ohwaki T, Yoshimoto T, Wada S. Effects of IL-23 and IL-27 on osteoblasts and osteoclasts: inhibitory effects on osteoclast differentiation. Journal of bone and mineral metabolism. 2007; 25:277-285.

8. Fox SW, Chow JW. Nitric oxide synthase expression in bone cells. Bone. 1998; 23:1-6.

9. Koo KH, Lee JS, Lee YJ, Kim KJ, Yoo JJ, Kim HJ. Endothelial nitric oxide synthase gene polymorphisms in patients with nontraumatic femoral head osteonecrosis. Journal of orthopaedic research. 2006; 24:1722-1728.

10. Athanasoulia AP, Sievers C, Ising M, Brockhaus AC, Yassouridis A, Stalla GK, Uhr M. Polymorphisms of the drug transporter gene ABCB1 predict side effects of treatment with cabergoline in patients with PRL adenomas. European journal of endocrinology/European Federation of Endocrine Societies. 2012; 167:327-335.

11. Fromm MF. Importance of P-glycoprotein for drug disposition in humans. European journal of clinical investigation. 2003; 33:6-9.

12. Sakaeda T, Nakamura T, Okumura K. Pharmacogenetics of MDR1 and its impact on the pharmacokinetics and pharmacodynamics of drugs. Pharmacogenomics. 2003; 4:397-410.

13. Sanchez E, Rueda B, Callejas JL, Sabio JM, OrtegoCenteno N, Jimenez-Alonso J, Lopez-Nevot MA, Martin J. Analysis of interleukin-23 receptor (IL23R) gene polymorphisms in systemic lupus erythematosus. Tissue antigens. 2007; 70:233-237.

14. Kim TH, Hong JM, Oh B, Cho YS, Lee JY, Kim HL, Lee JE, Ha MH, Park EK, Kim SY. Association of polymorphisms in the Interleukin 23 receptor gene with osteonecrosis of femoral head in Korean population. Experimental \& molecular medicine. 2008; 40:418-426.

15. Chao YC, Wang SJ, Chu HC, Chang WK, Hsieh TY. Investigation of alcohol metabolizing enzyme genes in Chinese alcoholics with avascular necrosis of hip joint, pancreatitis and cirrhosis of the liver. Alcohol and alcoholism. 2003; 38:431-436.

16. Xue Y, Zhao ZQ, Hong D, Zhang HJ, Chen HX, Fan SW. MDR1 gene polymorphisms are associated with glucocorticoid-induced avascular necrosis of the femoral head in a Chinese population. Genetic testing and molecular biomarkers. 2014; 18:196-201.

17. Wang Y, Li X, Gao Y, Li Z, Yu L, Meng Q, Sun L, Wang J. Genetic polymorphisms of CYP3A4 among Chinese patients with steroid-induced osteonecrosis of the femoral head. Medicine. 2016; 95:e5332.

18. Lagumdzija A, Ou G, Petersson M, Bucht E, Gonon A, Pernow Y. Inhibited anabolic effect of insulin-like growth factor-I on stromal bone marrow cells in endothelial nitric oxide synthase-knockout mice. Acta physiologica Scandinavica. 2004; 182:29-35.

19. Guler S, Gurkan H, Tozkir H, Turan N, Celik Y. An Investigation of the Relationship between the eNOS Gene Polymorphism and Diagnosed Migraine. Balkan journal of medical genetics. 2014; 17:49-59.

20. Gruber HJ, Bernecker C, Lechner A, Weiss S, WallnerBlazek M, Meinitzer A, Hobarth G, Renner W, Fauler G, Horejsi R, Fazekas F, Truschnig-Wilders M. Increased nitric oxide stress is associated with migraine. Cephalalgia: an international journal of headache. 2010; 30:486-492.

21. Casas JP, Cavalleri GL, Bautista LE, Smeeth L, Humphries SE, Hingorani AD. Endothelial nitric oxide synthase gene polymorphisms and cardiovascular disease: a HuGE review. American journal of epidemiology. 2006; 164:921-935.

22. Glueck CJ, Glueck HI, Welch M, Freiberg R, Tracy T, Hamer T, Stroop D. Familial idiopathic osteonecrosis mediated by familial hypofibrinolysis with high levels of plasminogen activator inhibitor. Thrombosis and haemostasis. 1994; 71:195-198.

23. Zhao L, Li K, Li W, Yang Z. Association between the C3435T polymorphism of ABCB1/MDR1 gene (rs1045642) and colorectal cancer susceptibility: a meta-analysis based on 11,339 subjects. Tumour biology: the journal of the International Society for Oncodevelopmental Biology and Medicine. 2013; 34:1949-1957.

24. Siegsmund M, Brinkmann U, Schaffeler E, Weirich G, Schwab M, Eichelbaum M, Fritz P, Burk O, Decker J, Alken P, Rothenpieler U, Kerb R, Hoffmeyer S, Brauch H. Association of the P-glycoprotein transporter MDR1(C3435T) polymorphism with the susceptibility to renal epithelial tumors. Journal of the American Society of Nephrology. 2002; 13:1847-1854.

25. Kim RB, Leake BF, Choo EF, Dresser GK, Kubba SV, Schwarz UI, Taylor A, Xie HG, McKinsey J, Zhou S, Lan LB, Schuetz JD, Schuetz EG, Wilkinson GR. Identification of functionally variant MDR1 alleles among European Americans and African Americans. Clinical pharmacology and therapeutics. 2001; 70:189-199.

26. Tan ZY, Bealgey KW, Fang Y, Gong YM, Bao S. Interleukin-23: immunological roles and clinical implications. The international journal of biochemistry \& cell biology. 2009; 41:733-735.

27. Abu-Shakra M, Buskila D, Shoenfeld Y. Osteonecrosis in patients with SLE. Clinical reviews in allergy \& immunology. 2003; 25:13-24.

28. Su Q, Wang Y, Zhao J, Ma C, Wu T, Jin T, Xu J. Polymorphisms of PRLHR and HSPA12A and risk of 
gastric and colorectal cancer in the Chinese Han population. BMC gastroenterology. 2015; 15:107.

29. Lee HJ, Choi SJ, Hong JM, Lee WK, Baek JI, Kim SY, Park EK, Kim SY, Kim TH, Kim UK. Association of a polymorphism in the intron 7 of the SREBF1 gene with osteonecrosis of the femoral head in Koreans. Annals of human genetics. 2009; 73:34-41.

30. Gabriel S, Ziaugra L, Tabbaa D. SNP genotyping using the Sequenom MassARRAY iPLEX platform. Current protocols in human genetics. 2009; Chapter 2:Unit 212.
31. Thomas RK, Baker AC, Debiasi RM, Winckler W, Laframboise T, Lin WM, Wang M, Feng W, Zander T, MacConaill L, Lee JC, Nicoletti R, Hatton C, et al. Highthroughput oncogene mutation profiling in human cancer. Nature genetics. 2007; 39:347-351.

32. Adamec C. [Example of the Use of the Nonparametric Test. Test X2 for Comparison of 2 Independent Examples]. Ceskoslovenske zdravotnictvi. 1964; 12:613-619.

33. Bland JM, Altman DG. Statistics notes. The odds ratio. Bmj. 2000; 320:1468. 